\title{
Case Report \\ Unilateral Subconjunctival and Retrobulbar Hemorrhage Secondary to Brodifacoum Toxicity in a Dog
}

\author{
Sonia E. Kuhn and Diane V. H. Hendrix \\ Department of Small Animal Clinical Sciences, College of Veterinary Medicine, University of Tennessee, 2407 River Drive, \\ Knoxville, TN 37996, USA \\ Correspondence should be addressed to Sonia E. Kuhn; skuhn3@utk.edu
}

Received 31 January 2013; Accepted 28 February 2013

Academic Editors: A. F. Koutinas and J. S. Munday

Copyright (C) 2013 S. E. Kuhn and D. V. H. Hendrix. This is an open access article distributed under the Creative Commons Attribution License, which permits unrestricted use, distribution, and reproduction in any medium, provided the original work is properly cited.

\begin{abstract}
An 8-year-old spayed female mixed-breed dog was presented for an acute onset of bleeding around the left eye. Mild exophthalmos and massive subconjunctival hemorrhage on the globe and nictitating membrane were present in the left eye. Retrobulbar hemorrhage was suspected, and pain was implied on opening of the mouth because the patient resisted and vocalized. No other abnormalities were found on ophthalmic or physical examination. Further questioning of the owner confirmed potential brodifacoum ingestion, and prothrombin time and partial thromboplastin time were both markedly elevated. Treatment with oral vitamin $\mathrm{K}_{1}$ was implemented, and the subconjunctival hemorrhage was significantly improved within a few days of instituting treatment. All clinical signs of coagulopathy were completely resolved within 4 weeks of presentation. Coagulopathy secondary to brodifacoum ingestion can manifest as severe unilateral bulbar and nictitating membrane subconjunctival hemorrhage and exophthalmos due to retrobulbar hemorrhage without other clinical signs.
\end{abstract}

\section{Introduction}

Intraocular and periocular bleeding can occur with primary disease of the globe and adnexa or as manifestations of systemic disease. Clinical signs are hyphema and hemorrhage of nearly any aspect of the eye, including the uvea, vitreous, retina, subretinal space, conjunctiva, subconjunctival, and retrobulbar space. Periocular and intraocular hemorrhages are most commonly associated with uveitis or retinal detachment [1] caused by infectious diseases, including systemic fungal [2] and rickettsial diseases [3-5]; immune-mediated diseases such as uveodermatologic syndrome [6]; bleeding and vascular disorders such as hypertension [7, 8], thrombocytopenia $[9,10]$, anemia [11] and coagulopathy [1]; neoplasia [1, 12]; diabetes mellitus [13]; and hyperviscosity syndrome from multiple myeloma $[8,14]$ and polycythemia vera [8]. Additionally, persistent hyperplastic primary vitreous [15], retinal dysplasia, preiridal fibrovascular membrane formation [16], and blunt or penetrating trauma $[1,17]$ can also cause intraocular hemorrhage. Retrobulbar hemorrhage occurs because of trauma or coagulopathy and can cause exophthalmos [18-21].
Conjunctival or scleral hemorrhage in dogs usually occurs focally as petechiae from a primary hemostatic disorder, which is typically due to thrombocytopenia. Although subconjunctival hemorrhage is uncommon, it can occur secondary to coagulopathy, trauma, and vasculitis [21]. It has also been reported to occur with Rocky Mountain spotted fever [3] and scleral rupture [17]. The aim of this report is to describe an atypical presentation of brodifacoum rodenticide toxicity where the only clinical signs were unilateral subconjunctival and retrobulbar hemorrhages.

\section{Case Presentation}

An 8-year-old spayed female mixed-breed dog was presented for an acute onset of bleeding around the left eye. The bleeding began 6 hours prior to presentation and was progressively worsening. The dog had been alone in a fenced yard for most of the day. Her activity level was appropriate, and thirst and appetite were normal. No known trauma had occurred. Prior medical history was unremarkable. The dog had been sprayed by a skunk (Mephitis mephitis) 2 weeks prior to 
presentation but did not seem to suffer any ill effects from that incident. Appropriate vaccinations were current, and she was not receiving any medication aside from heartworm and flea prophylaxis.

On ophthalmic examination, direct and consensual pupillary light responses were normal in both eyes (OU). A menace response was present $\mathrm{OU}$, and the dog exhibited behavior consistent with vision bilaterally. The Schirmer tear test 1 (Schirmer tear test strips, Merck Animal Health) showed normal tear production in the right eye (OD) at $19 \mathrm{~mm} / \mathrm{min}$ and was not evaluated in the left eye (OS) due to the bleeding (reference range $>15 \mathrm{~mm} / \mathrm{min}$ ). A drop of $0.5 \%$ proparacaine hydrochloride ophthalmic solution (Alcaine, Alcon Laboratories) was applied topically OU, and applanation tonometry (Tono-Pen Vet, Reichert, Inc.) was performed. Intraocular pressure was $15 \mathrm{mmHg} \mathrm{OU}$ (reference range $6-24 \mathrm{mmHg}$ ) [22]. Massive hemorrhage was present beneath the bulbar conjunctiva for $360^{\circ}$ around the left globe as well as beneath the palpebral and bulbar conjunctiva of the nictitating membrane OS (Figure 1(a)). Mild serosanguinous discharge was also present OS. The left globe was mildly exophthalmic (Figure 1(b)), and retropulsion seemed to cause moderate pain. The left eyelids were mildly swollen, and palpation of the left bony orbit was unremarkable. Slit-lamp biomicroscopy (Kowa SL-15, Kowa) and indirect ophthalmoscopy (20 D handheld lens, Volk Optical Inc.; Vantage Plus Wireless Headset, Keeler Instruments Inc.) were performed OU. Examination of the left and right anterior and posterior segments were unremarkable aside from prominent suture tip opacities in the lens OD. Nuclear sclerosis was noted OU. No evidence of bleeding was detected in or around the right eye.

The only abnormality found on physical examination was pain on opening of the mouth, as the patient resisted and vocalized during this maneuver. Temperature, pulse, and respiratory rate were within normal limits. The patient was bright, alert, and responsive. The mucous membranes were pink with a capillary refill time of $<2$ seconds. Thoracic auscultation and abdominal palpation were unremarkable. Neither petechiae nor ecchymoses were found, and no evidence of trauma was present.

Initial differential diagnoses for the subconjunctival hemorrhage OS were coagulopathy and trauma. Upon further questioning of the owner, it was confirmed that brodifacoum rodenticide (d-CON rat and mice bait pellets, Reckitt Benckiser) had been placed in the yard occupied by the dog 10 days prior to presentation. Prothrombin time (PT) and partial thromboplastin time (PTT) were tested. The PT was prolonged beyond quantification (reference range 6.88.7 seconds), and PTT was markedly prolonged at 146.5 seconds (reference range 14.5-25.6 seconds). A complete blood count, including platelet estimation, was within normal limits. Total protein and hematocrit were both normal at $7.0 \mathrm{~g} / \mathrm{dL}$ (reference range 5.7-7.9 $\mathrm{g} / \mathrm{dL}$ ) and 55.7\% (reference range $41-60 \%)$, respectively. Pleural fluid was not seen on thoracic ultrasound, and a small amount of free abdominal fluid was seen around the bladder on abdominal ultrasound. This fluid was presumed to be blood, but abdominocentesis for confirmation was not performed due to the coagulopathy.
The patient was admitted to the hospital for overnight monitoring and treatment. A subcutaneous injection of $93 \mathrm{mg}(5 \mathrm{mg} / \mathrm{kg})$ vitamin $\mathrm{K}_{1}$ was given at admission and was repeated 12 hours later. Oral treatment with $25 \mathrm{mg}(1.3 \mathrm{mg} / \mathrm{kg})$ of vitamin $\mathrm{K}_{1}$ twice daily was then instituted for 4 weeks. The left eye was lubricated with artificial tears ophthalmic ointment (15\% mineral oil with $83 \%$ white petrolatum, Rugby Laboratories) four times daily to prevent exposure keratitis secondary to exophthalmos and lagophthalmos from nictitating membrane and eyelid swelling. Exercise restriction to prevent additional bleeding was also instituted.

Two days later, PT and PTT were within normal limits at 7.7 seconds and 17.8 seconds, respectively. The subconjunctival hemorrhage and eyelid swelling OS were markedly improved (Figure 2(a)). Artificial tears ointment was discontinued since the globe was no longer exophthalmic. The Schirmer tear test 1 was within normal limits OU at $15 \mathrm{~mm} / \mathrm{min}$ OD and $21 \mathrm{~mm} / \mathrm{min}$ OS. Applanation tonometry showed normal intraocular pressures OU of $8 \mathrm{mmHg}$ OD and $10 \mathrm{mmHg}$ OS. Examination of the anterior segment and fundus was unchanged OU.

The patient was reevaluated 1 month after initial presentation. Oral vitamin $\mathrm{K}_{1}$ had been discontinued 2 days prior to examination. The PT was within normal limits at 7.4 seconds, and PTT was slightly prolonged at 26.4 seconds. The subconjunctival and periocular hemorrhage OS was fully resolved (Figure 2(b)). Examination of the anterior and posterior segments was unchanged OU from the initial presentation. Oral vitamin $K_{1}$ therapy was not reinstituted since the coagulopathy had resolved. Communication with the owner 3 months later revealed the dog to be completely asymptomatic and free of any remaining detectable abnormalities.

\section{Discussion}

Ocular lesions have been documented with anticoagulant rodenticide exposure but are rarely mentioned in texts that discuss clinical signs of this toxicity. This may be because ocular lesions are uncommon relative to other signs, or because they are usually mild in comparison to the more life-threatening hemorrhage that typically occurs, such as hemothorax. Hyphema as well as scleral and subconjunctival hemorrhage has been previously reported with anticoagulant rodenticides [1, 21, 23-25]. The previously reported scleral hemorrhage may have been referring to subconjunctival hemorrhage, though photographs of the lesions were not provided $[23,26]$. Concurrent exophthalmos or suspected retrobulbar hemorrhage was not mentioned in those cases where scleral hemorrhage was noted, and one of the cases had other obvious concurrent clinical signs of coagulopathy [26]. In one retrospective study evaluating clinical signs of coagulopathy due to anticoagulant rodenticide, scleral hemorrhage was an uncommon finding that was seen in only 3 of 52 cases [23]. Whether this hemorrhage occurred with or without other clinical signs of coagulopathy was not discussed.

Massive subconjunctival hemorrhage of the nictitating membrane has not been previously documented as a clinical 


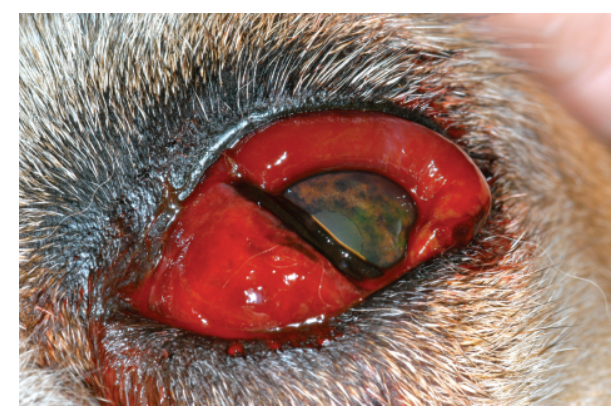

(a)

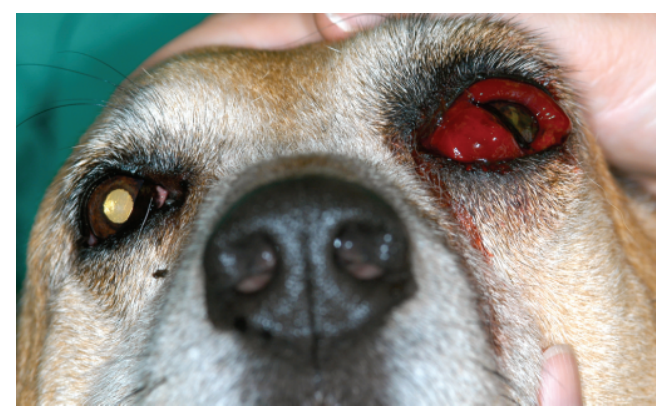

(b)

FIGURE 1: At initial presentation, severe subconjunctival hemorrhage of the globe, and nictitating membrane were present around the left eye. Mild exophthalmos, frank bleeding, and serosanguinous discharge were also seen. These were the only clinical signs of coagulopathy secondary to brodifacoum ingestion.

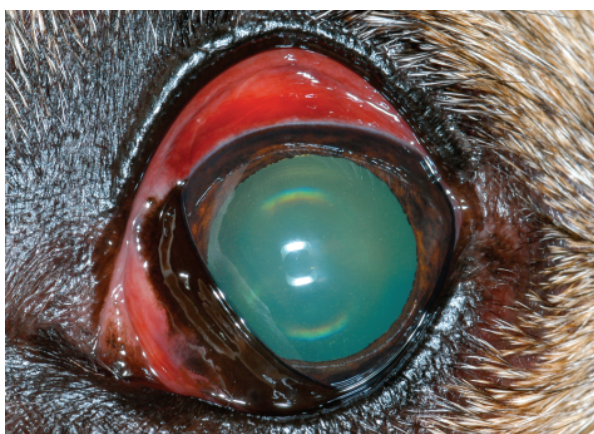

(a)

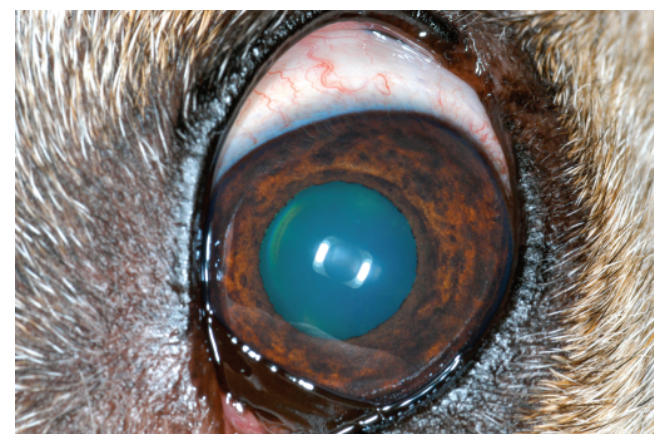

(b)

Figure 2: (a) Two days after therapy with oral vitamin $\mathrm{K}_{1}$, the exophthalmos was resolved and the subconjunctival hemorrhage greatly improved. (b) The subconjunctival hemorrhage was resolved after treatment with oral vitamin $\mathrm{K}_{1}$ for 4 weeks.

sign of anticoagulant rodenticide toxicity, though exophthalmos secondary to retrobulbar hemorrhage is known to occur [19-21]. Retrobulbar hematoma secondary to warfarin toxicity has also been reported to cause exophthalmos in humans [27]. Other causes of acquired coagulopathy that could cause subconjunctival or retrobulbar hemorrhage in dogs are severe liver disease; vasculitis; autoimmune disease directed against a coagulation factor; disseminated intravascular coagulation (DIC); anticoagulant therapy; or low levels of vitamin $\mathrm{K}$ secondary to obstructive hepatopathy, malabsorption, or low dietary vitamin $\mathrm{K}[21,28,29]$.

Bleeding disorders can be classified as diseases that affect fibrinolysis, primary hemostasis, and secondary hemostasis. Fibrinolysis is responsible for fibrin clot dissolution and depends on the conversion of plasminogen to plasmin [29]. Clot dissolution forms fibrin degradation products, which subsequently are removed from circulation by the liver. Accumulation of these products causes bleeding tendencies by interfering with platelet function and thrombin inhibition [29]. Primary hemostasis seals injured blood vessels with the creation of the primary hemostatic plug via the interactions of platelets and endothelium. Platelet or endothelial diseases, such as thrombocytopenia, thrombocytopathia, and vasculopathies, cause primary hemostatic disorders [29].

Secondary hemostasis has traditionally been defined using a cascade model where the intrinsic and extrinsic enzymatic pathways converge into a common pathway that results in the conversion of fibrinogen to fibrin [29]. The fibrin produced by secondary hemostasis solidifies the primary hemostatic plug. Factors VIII, IX, XI, and XII are involved in the intrinsic pathway, while tissue factor and factor VII constitute the extrinsic pathway. The common pathway involves factor $\mathrm{X}$ and the conversion of prothrombin to thrombin (factor II), which ultimately leads to the cleavage of fibrin from fibrinogen. While PT evaluates the extrinsic and common pathways, PTT evaluates the intrinsic and common pathways. More recently, coagulation has been described with a cell-based model, which explains in vivo deficiencies seen with the cascade model [30]. The cell-based model views coagulation as occurring in distinct yet overlapping phases rather than separate enzymatic pathways [30]. It also accounts for the role of cell surfaces in fibrin formation as well as the additional functions of coagulation proteins beyond the coagulation cascade [30]. The liver produces most of the coagulation factors necessary for secondary hemostasis [29]. Factors II, VII, IX, and X are known as vitamin Kdependent coagulation factors since they contain glutamyl residues that must be activated with carboxylation, which requires reduced vitamin $K_{1}$ as a cofactor [28, 31]. These glutamyl residues allow for binding of the coagulation protein to a cell membrane surface via calcium binding, and calcium binding cannot occur unless carboxylation occurs [30]. 
Carboxylation results in the oxidation of vitamin $\mathrm{K}_{1}$, and the enzyme vitamin $K_{1}$ epoxide reductase is necessary to reduce vitamin $K_{1}$ back to its active form so that it can be recycled to activate additional coagulation factors [28]. Disorders of secondary hemostasis are due to decreased concentrations of or ineffective coagulation factors and result in coagulopathies.

Anticoagulant rodenticides, as described in this case, cause coagulopathy by depletion of vitamin $\mathrm{K}$-dependent coagulation factors via inhibition of vitamin $K_{1}$ epoxide reductase [20, 31]. Warfarin and pindone are first-generation anticoagulants, while brodifacoum, bromadiolone, and diphacinone are second-generation anticoagulants [31]. The second-generation anticoagulants have longer half-lives, less drug-acquired resistance, and increased potency [20, 31, 32]. Clinical signs of coagulopathy secondary to brodifacoum manifest 2 to 5 days after ingestion and vary based on the location and severity of the hemorrhage [20, 29, 31]. The most common are dyspnea, lethargy, coughing, hemoptysis, pale mucous membranes, and tachycardia [23, 24]. Bleeding typically occurs into body cavities causing hemothorax, hemoabdomen, and retroperitoneal hemorrhage [20, 23]. Less frequent signs are melena, hematochezia, prolonged bleeding at injection sites, epistaxis, gingival bleeding, and neurologic signs $[23,24]$. Case reports of atypical presentations of coagulopathy due to anticoagulant rodenticide include lameness from hemarthrosis [33], pericardial effusion [34], hematometra [35], hydronephrosis secondary to blood clots in the urinary bladder [36], tracheal obstruction [37], and submucosal gastric hemorrhage [38].

The earliest laboratory abnormality detected after secondgeneration anticoagulant rodenticide toxicity is elevation of the proteins induced by vitamin $\mathrm{K}$ absence or antagonism [31]. Prolongation of PT occurs within 36-72 hours of ingestion and precedes prolongation of PTT because of the short half-life of factor VII $[20,29]$. Neither PTT nor activated clotting time is prolonged until greater than 72 hours after ingestion [20]. Decontamination of the patient is not indicated after signs of coagulopathy have developed since the toxin was consumed several days before examination. Patients with severe hemorrhage and subsequent anemia may require transfusions of whole blood or fresh frozen plasma to provide red bloods cells and coagulation factors to halt bleeding. Oral vitamin $\mathrm{K}_{1}$ at $1.25-2.5 \mathrm{mg} / \mathrm{kg}$ twice daily for 4 weeks is needed to resolve coagulopathy caused by secondgeneration anticoagulants [31]. Therapy is needed for only 2 weeks for first-generation anticoagulants and for 3 weeks with bromadiolone since these agents are shorter acting [20]. Absorption of oral vitamin $K_{1}$ is improved if given with a fatty meal [20]. A subcutaneous loading dose of 2.5$3.3 \mathrm{mg} / \mathrm{kg}$ of vitamin $\mathrm{K}_{1}$ is recommended by some clinicians and discouraged by others $[19,20,31,39,40]$. Injections can cause additional bleeding and hematoma formation and are not more bioavailable than the oral formulation [19]. Intravenous administration is not recommended due to the risk of anaphylaxis [39]. Treatment should be discontinued for 2 days before repeated testing of PT to ensure that the patient is able to produce active coagulation factors without supplementation. If $\mathrm{PT}$ is still prolonged after discontinuation of vitamin $\mathrm{K}_{1}$, then supplementation is continued for another week and the patient is then retested [20].

This case is an unusual presentation of coagulopathy secondary to brodifacoum ingestion in that unilateral subconjunctival and retrobulbar hemorrhages were the only apparent clinical signs. Subconjunctival hemorrhage can occur because of scleral or conjunctival bleeding, or anterior migration of retrobulbar blood [21, 25]. Retrobulbar hemorrhage was presumed because of the exophthalmos and severe subconjunctival hemorrhage OS, though this was not confirmed with imaging or sample collection. While fresh frozen plasma is usually indicated for patients with active bleeding, it was not administered in this case since no other significant detectable body cavity effusion had occurred at the time of diagnosis and the patient was not anemic. Anemia is present in the majority of coagulopathic dogs after rodenticide ingestion and can be seen in $83 \%$ of cases [24]. Monitoring was continued for the first 24 hours of vitamin $K_{1}$ therapy so that fresh frozen plasma could have been administered if necessary. This patient had complete resolution of all clinical signs with oral vitamin $K_{1}$ supplementation, and no permanent adverse effects occurred to the globe or periocular structures OS. The mild prolongation of PTT seen in this patient at the conclusion of vitamin $\mathrm{K}_{1}$ therapy was not indicative of an unresolved coagulopathy since the PT was normal. Mild elevations of PTT are often clinically insignificant, and PT is a more sensitive indicator of coagulopathy secondary to vitamin K deficiency [29].

As with other reports of atypical presentations of coagulopathy secondary to anticoagulant rodenticide ingestion, this case further reinforces that patients with this syndrome can present with bleeding in nearly any location. Prognosis is excellent as long as a prompt diagnosis is made and proper treatment instituted. An incorrect initial diagnosis occurs in up to $25 \%$ of cases of coagulopathy due to anticoagulant rodenticide toxicity, which can be life threatening because treatment is delayed and hemorrhage continues [24]. Patient history can also be misleading, as owners denied pet exposure to anticoagulant rodenticide in over $50 \%$ of confirmed cases in one study [41]. The massive unilateral subconjunctival hemorrhage in this case allowed for rapid diagnosis of the patient's coagulopathy before more serious bleeding and anemia occurred. In summary, anticoagulant rodenticides should be strongly considered in cases of unilateral subconjunctival hemorrhage of the globe and nictitating membrane or when retrobulbar hemorrhage is suspected.

\section{References}

[1] A. Trbolova, "Hyphema in dogs: 91 cases," Veterinary Ophthalmology, vol. 12, no. 1, pp. 61-70, 2009.

[2] K. N. Gelatt, L. D. McGill, and V. Perman, "Ocular and systemic cryptococcosis in a dog," Journal of the American Veterinary Medical Association, vol. 162, no. 5, pp. 370-375, 1973.

[3] M. G. Davidson, E. B. Breitschwerdt, M. P. Nasisse, and S. M. Roberts, "Ocular manifestations of Rocky Mountain spotted fever in dogs," Journal of the American Veterinary Medical Association, vol. 194, no. 6, pp. 777-781, 1989. 
[4] A. A. Komnenou, M. E. Mylonakis, V. Kouti et al., "Ocular manifestations of natural canine monocytic ehrlichiosis (Ehrlichia canis): a retrospective study of 90 cases," Veterinary Ophthalmology, vol. 10, no. 3, pp. 137-142, 2007.

[5] J. F. Wilson, "Ehrlichia platys in a Michigan dog," Journal of the American Animal Hospital Association, vol. 28, pp. 381-383, 1992.

[6] J. L. Laus, M. G. Sousa, V. P. Cabral, F. V. Mamede, and M. Tinucci-Costa, "Uveodermatologic syndrome in a Brazilian Fila dog," Veterinary Ophthalmology, vol. 7, no. 3, pp. 193-196, 2004.

[7] N. L. LeBlanc, R. L. Stepien, and E. Bentley, "Ocular lesions associated with systemic hypertension in dogs: 65 cases (20052007)," Journal of the American Veterinary Medical Association, vol. 238, no. 7, pp. 915-921, 2011.

[8] I. F. Lane, S. M. Roberts, and M. R. Lappin, "Ocular manifestations of vascular disease: hypertension, hyperviscosity and hyperlipidemia," Journal of the American Animal Hospital Association, vol. 29, no. 1, pp. 28-36, 1993.

[9] S. K. O’Marra, A. M. Delaforcade, and S. P. Shaw, “Treatment and predictors of outcome in dogs with immune-mediated thrombocytopenia," Journal of the American Veterinary Medical Association, vol. 238, no. 3, pp. 346-352, 2011.

[10] I. Aubert, M. Carrier, M. Desnoyers, and L. Breton, "A case of bilateral hyphema secondary to an immune-mediated thrombocytopenia in a dog," Medecin Veterinaire Du Quebec, vol. 27, no. 3, pp. 103-108, 1997.

[11] C. L. Cullen and A. A. Webb, "Ocular manifestations of systemic diseases part 1: the dog," in Veterinary Ophthalmology, K. N. Gelatt, Ed., pp. 1470-1537, Blackwell, Ames, Iowa, USA, 4th edition, 2004.

[12] S. G. Krohne, N. M. Henderson, R. C. Richardson, and W. A. Vestre, "Prevalence of ocular involvement in dogs with multicentric lymphoma: prospective evaluation of 94 cases," Veterinary and Comparative Ophthalmology, vol. 4, pp. 127-135, 1994.

[13] M. P. Landry, I. P. Herring, and D. L. Panciera, "Funduscopic findings following cataract extraction by means of phacoemulsification in diabetic dogs: 52 cases (1993-2003)," Journal of the American Veterinary Medical Association, vol. 225, no. 5, pp. 709-716, 2004.

[14] D. V. H. Hendrix, K. N. Gelatt, P. J. Smith, D. E. Brooks, C. J. G. Whittaker, and N. T. Chmielewski, "Ophthalmic disease as the presenting complaint in five dogs with multiple myeloma," Journal of the American Animal Hospital Association, vol. 34, no. 2, pp. 121-128, 1998.

[15] A. Bayón, M. C. Tovar, M. J. Fernández Del Palacio, and A. Agut, "Ocular complications of persistent hyperplastic primary vitreous in three dogs," Veterinary Ophthalmology, vol. 4, no. 1, pp. 35-40, 2001.

[16] B. H. Grahn and R. L. Peiffer, "Fundamentals of veterinary ophthalmic pathology," in Veterinary Ophthalmology, K. N. Gelatt, Ed., pp. 355-437, Blackwell, Ames, Iowa, USA, 4th edition, 2004.

[17] A. Rampazzo, C. Eule, S. Speier, P. Grest, and B. Spiess, "Scleral rupture in dogs, cats, and horses," Veterinary Ophthalmology, vol. 9, no. 3, pp. 149-155, 2006.

[18] B. M. Spiess, "Diseases and surgery of the canine orbit," in Veterinary Ophthalmology, K. N. Gelatt, Ed., pp. 539-562, Blackwell, Ames, Iowa, USA, 4th edition, 2004.

[19] C. Means, "Anticoagulant rodenticide toxicosis," in Clinical Veterinary Advisor Dogs and Cats, E. Coté, Ed., pp. 76-77, Mosby, St. Louis, Mo, USA, 1st edition, 2007.
[20] C. DeClementi and B. R. Sobczak, "Common rodenticide toxicoses in small animals," Veterinary Clinics of North America Small Animal Practice, vol. 42, pp. 349-360, 2012.

[21] C. L. Martin, "Conjunctiva and third eyelid," in Ophthalmic Disease in Veterinary Medicine, C. L. Martin, Ed., pp. 183-218, Manson, London, UK, 1st edition, 2005.

[22] H. E. Klein, S. G. Krohne, G. E. Moore, A. S. Mohamed, and J. Stiles, "Effect of eyelid manipulation and manual jugular compression on intraocular pressure measurement in dogs," Journal of the American Veterinary Medical Association, vol. 238, no. 10, pp. 1292-1295, 2011.

[23] B. Haines, "Anticoagulant rodenticide ingestion and toxicity: a retrospective study of 252 canine cases," Australian Veterinary Practitioner, vol. 38, no. 2, pp. 38-50, 2008.

[24] S. E. Sheafor and C. Guillermo Couto, "Anticoagulant rodenticide toxicity in 21 dogs," Journal of the American Animal Hospital Association, vol. 35, no. 1, pp. 38-46, 1999.

[25] C. L. Martin, "Ocular manifestations of systemic disease part 1 the dog," in Veterinary Ophthalmology, K. N. Gelatt, Ed., pp. 1401-1448, Lippincott Williams \& Wilkins, Philadelphia, Pa, USA, 3rd edition, 1999.

[26] M. Schaer and C. Henderson, "Suspected warfarin toxicosis in a dog," Journal of the American Veterinary Medical Association, vol. 176, no. 6, pp. 535-536, 1980.

[27] D. Thompson, C. Stanescu, P. Pryor, and B. Laselle, "Retrobulbar hematoma from warfarin toxicity and the limitations of bedside ocular sonography," Western Journal of Emergency Medicine, vol. 11, no. 2, pp. 208-210, 2010.

[28] D. C. Baker, "Dgnosis of disorders of hemostasis," in Veterinary Hematology and Clinical Chemistry, M. A. Thrall, Ed., pp. 179196, Blackwell, Ames, Iowa, USA, 2nd edition, 2006.

[29] S. G. Hackner, "Bleeding disorders," in Small Animal Critical Care Medicine, D. C. Silverstein and K. Hopper, Eds., pp. 507514, Elsevier Saunders, St. Louis, Mo, USA, 1st edition, 2009.

[30] S. A. Smith, “The cell-based model of coagulation," Journal of Veterinary Emergency and Critical Care, vol. 19, no. 1, pp. 3-10, 2009.

[31] R. M. DuFort and L. Matros, "Acquired coagulopathies," in Textbook of Veterinary Internal Medicine, S. J. Ettinger and E. C. Feldman, Eds., pp. 1933-1937, Elsevier Saunders, St. Louis, Mo, USA, 6th edition, 2005.

[32] M. Lund, "Comparative effect of the three rodenticides warfarin, difenacoum and brodifacoum on eight rodent species in short feeding periods," Journal of Hygiene, vol. 87, no. 1, pp. 101107, 1981.

[33] J. R. Bellah and J. P. Weigel, "Hemarthrosis secondary to suspected warfarin toxicosis in a dog," Journal of the American Veterinary Medical Association, vol. 182, no. 10, pp. 1126-1127, 1983.

[34] D. J. Petrus and A. Rosemary, "Pericardial effusion and cardiac tamponade secondary to brodifacoum toxicosis in a dog," Journal of the American Veterinary Medical Association, vol. 215, no. 5, pp. 647-648, 1999.

[35] S. L. Padgett, J. E. Stokes, R. L. Tucker, and L. G. Wheaton, "Hematometra secondary to anticoagulant rodenticide toxicity," Journal of the American Animal Hospital Association, vol. 34, no. 5, pp. 437-439, 1998.

[36] N. Hansen and C. Beck, "Bilateral hydronephrosis secondary to anticoagulant rodenticide intoxication in a dog," Journal of Veterinary Emergency and Critical Care, vol. 13, no. 2, pp. 103107, 2003. 
[37] T. L. Blocker and B. K. Roberts, "Acute tracheal obstruction associated with anticoagulant rodenticide intoxication in a dog," Journal of Small Animal Practice, vol. 40, no. 12, pp. 577580, 1999.

[38] S. L. Marks, T. L. Gieger, and J. Williams, "Presumptive intramural gastric hemorrhage secondary to rodenticide intoxication in a dog," Journal of Veterinary Emergency and Critical Care, vol. 11, no. 1, pp. 27-31, 2001.

[39] A. J. Brown and L. S. Waddell, "Rodenticides," in Small Animal Critical Care Medicine, D. C. Silverstein and K. Hopper, Eds., pp. 346-350, Elsevier Saunders, St. Louis, Mo, USA, 1st edition, 2009.

[40] B. J. Woody, M. J. Murphy, A. C. Ray, and R. A. Green, "Coagulopathic effects and therapy of brodifacoum toxicosis in dogs," Journal of Veterinary Internal Medicine, vol. 6, no. 1, pp. 23-28, 1992.

[41] L. W. Tseng, R. H. Poppenga, and D. Hughes, "Anticoagulant rodenticide toxicity and serum anticoagulant rodenticide concentrations in 43 dogs (1997-2000)," in Proceedings of the Seventh International Veterinary Emergency and Critical Care Symposium, p. 798, 2000. 

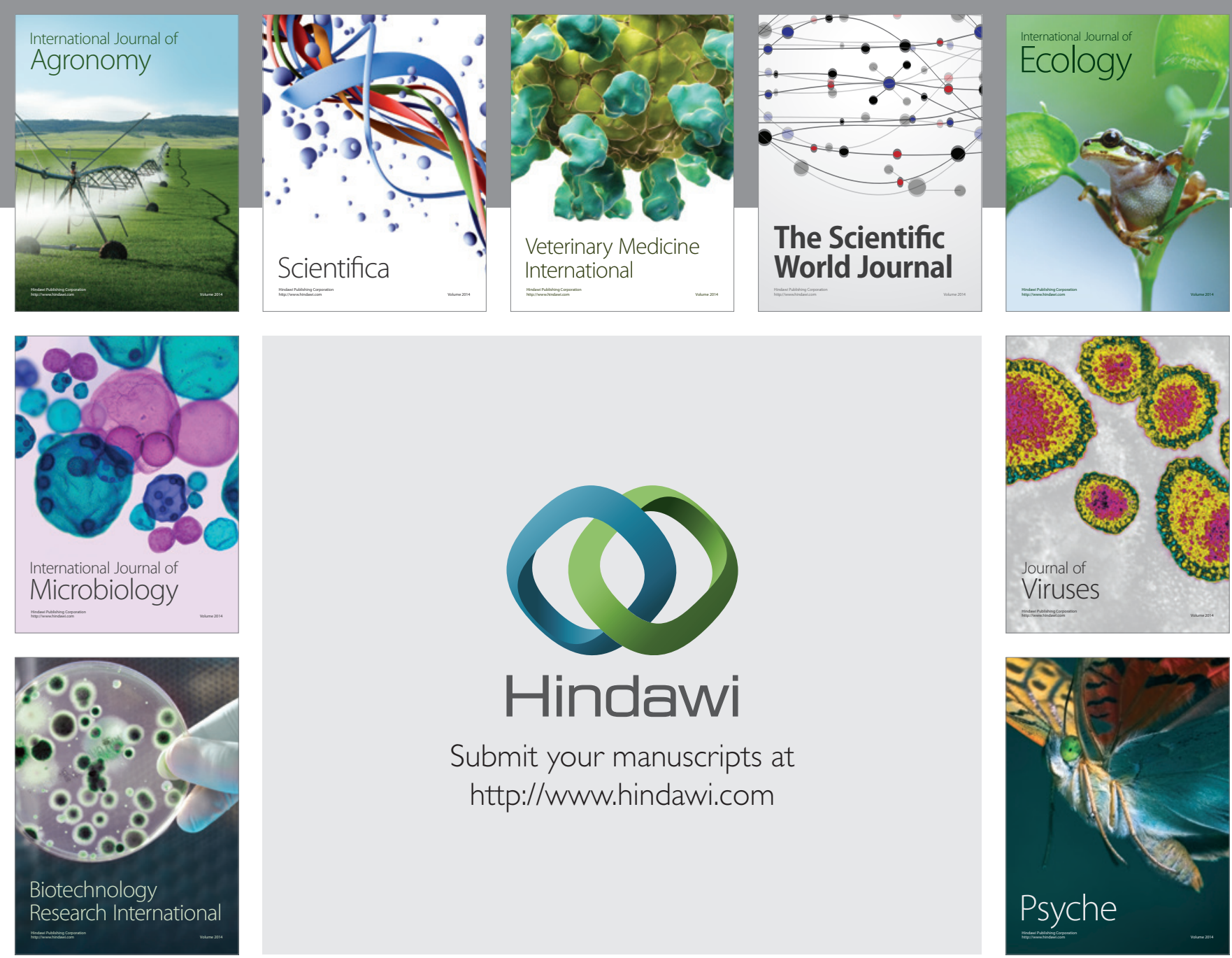

Submit your manuscripts at http://www.hindawi.com
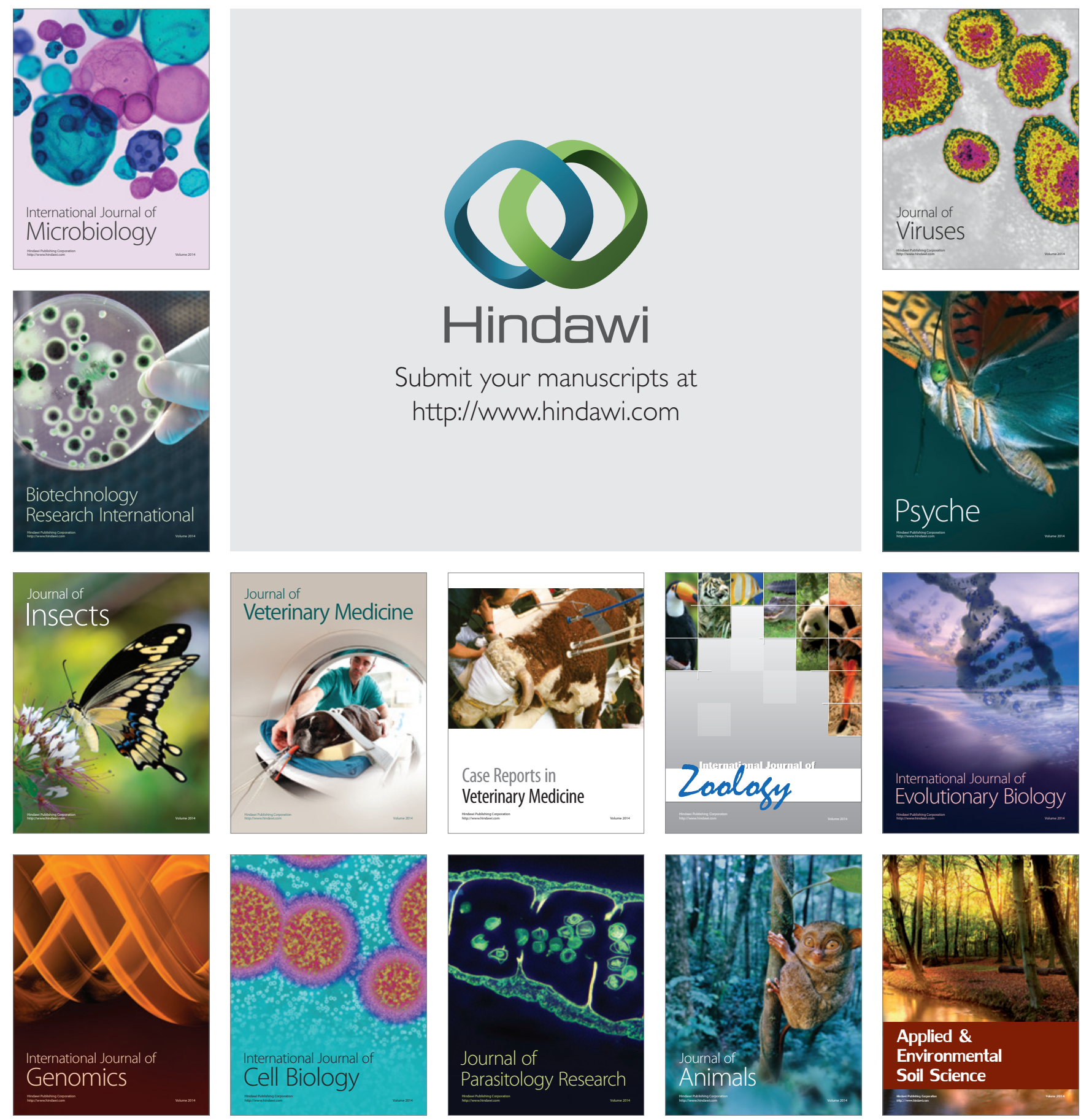